\section{Staphylococcal Infections.}

I have now discussed the main affections of the skin due to streptococcal infection; it remains to say a few words about staphylococcal infections. Although the Staphylococcus epidermidis albus is a constant inhabitant of the skin, pure infections by pyogenic staphylococci are not so common as is the case with pyogenic streptococci. It is probable, however, that most of the septic complications of ordinary moist dermatoses are due to staphylococci, but owing to the varied flora found under these conditions, it is difficult to apportion blame.

The following are the commonest staphylococcal lesions found in children :-

\section{Boclihart's Impetigo.}

This is a superficial pustulation of the hair follicles, a folliculitis, which is not very common by itself, but sometimes complicates septic dermatitis of the hairy regions. It is best seen in the condition called "pustular eczema of the scalp" already referred to. The pustules are very superficial and do not extend down the hair follicles to any depth; they usually give pure cultures of staph. pyogenes aureus. They are resistant to treatment and should not, as a rule, be treated with any ointment or greasy preparation. Spirit lotions with, weak antiseptics are most successful, such as 1 in 4000 perchloride of mercury in 60 per cent. alcohol, or liq. calcis sulphurat. $3 \mathrm{i}$., sp. vin. rect. ろii., glycerin 3ii., aq. ad ऊ viii. When much general dermatitis is present, lead or aluminium acetate lotion is better tolerated.

Vaccines are worth a trial in resistant cases, but are only occasionally helpful. Epilation by means of $X$ rays is necessary in some cases.

\section{Furunculosis.}

Boils in children are often complications of other infected skin conditions, and usually subside when the primary condition is treated. It is necessary, however, to treat each boil with proper surgical precautions, so as to prevent spread of infection. I am not personally in favour of early incision, but as soon as pus points, the boil should be pricked and the pus, usually quite a small amount, gently evacuated. Boric fomentations should never be applied as they tend to spread infection. Compresses of 1 in 4000 perchloride of mercury are the best dressings. No further treatment is necessary, unless further crops of boils develop. This is an indication that the patient has become hypersensitive to staphylococci, and general treatment should be adopted to try to raise the patient's resistance. If vaccines are given, they should be used with great caution and very small doses tried in the first instance, or the condition may become worse. General tonics are useful and sulphur may also be given internally, either as colloidal sulphur or calcium sulphide. Injections of colloidal manganese have proved useful in some casesc Boils in children, however, are rarely as trouble some as in adults, and treatment on general line $\$$ is usually quickly successful.

\section{Multiple Abscesses in Infants.}

Some debilitated infants develop crops of subs cutaneous abscesses, usually painless, which 理 cultured grow Staph. pyogenes aureus. These are apt to end fatally, unless dealt with in the earlo stages. The treatment is mainly surgical, evacuae tion of the pus and careful dressings being th\& important thing. The infant should be kept warm and put on a suitable diet, so as to increase its body-weight.

These are the commoner affections due to pyogenig organisms met with in infants and children; othes secondary infections can be dealt with on simila lines.

\section{BROMIDE ERUPTIONS.}

\section{BY}

H. HALDIN-DAVIS, M.D. Oxf., F.R.C.S. Excre DERMATOLOGIST TO THE ROYAL FREE HOSPITAL.

THese eruptions are of course well knowrg especially to physicians who are concerned wit the treatment of nervous diseases, above all wit? epilepsy. Their commonest manifestations ar: illustrated well by the name often employed to describe the appearances produced-i.e., " bromid acne," which emphasises the point that the salien characteristic of all cutaneous bromodermia is the production of pus. But there are several variac tions from the common type. In many cases the lesions are extensive and take on a somewha warty appearance, with pus exuding from a numbè of tiny openings. Such cases usually offer particular difficulty in diagnosis as they show thens selves under circumstances in which bromodermi is expected, although they are of practical impog tance as they interfere with the treatment of thes nervous condition for which the bromide is bein administered and may even cause its interruption Such cases are described as being of the "mycotic" or frambœsiform type of bromodermia, and a goode discussion of this type of eruption will be found in the May number of the British Journal of Dermatology, 1923, by Parkes Weber, who had some patients very severely affected under his carø, patients, in fact, in whom ulceration and granules matous tumour formation were so conspicuous that unless their condition had always varied exact $\vec{Q}$ with the exhibition or withdrawal of their medicin it would have been hard to believe that the bromide had caused the terrible cutaneous lesions fro which they suffered. A similar case was show by Sibley before the Dermatological Section of tige Royal Society of Medicine in 1917, and doubt 
cast on his diagnosis, but it was confirmed by the subsequent course of the case.

Bromodermia in infants nursed by epileptic mothers who are taking the drug is well known; there is a good illustration of such a case in Allbutt's "System of Medicine," Vol. IX. Much rarer is congenital bromodermia. I have, however, seen one such case, a female baby a week old, the daughter of a lady who, although not an epileptic, had, on the advice of a distinguished neurologist, taken five grains of bromide regularly throughout her pregnancy. The patient was sent to me by Dr. Margaret Rorke. It is noteworthy that the eruption did not appear until several days after the child was born. The explanation of the delay must be that for the production of the rash staphylococci are necessary, and these are not found in the healthy pregnant uterus. It appears that the action of bromide is to inhibit the natural bactericidal powers of the patient so far as staphylococci are concerned. This process of sensitisation having taken place in utero, the appearance of the rash followed very shortly after the entry of the infant into a very sepsis-ridden world. The only other case I have heard of similar in nature was one published by J. Langer in the Jahrbuch für Kinderheilkunde, 1922. Generally, bromodermia in infancy shows for the most part nodular lesions, rather than granulomatous or fungating tumours.

\section{Illustrative Cases.}

The really puzzling cases of bromodermia are those in which there is but a single lesion. Such cases are much rarer than those of the usual kind, and it is difficult to give the exact characteristics of the lesion, which varies greatly. In my personal experience I have only come across three cases of this kind.

One was in a girl aged 8 years, who was the daughter of an epileptic father and also suffered from fits herself, for which she took small but regular doses of the drug. She had a single ulcerative lesion, which took the shape of a narrow semicircular band on the outer aspect of the middle of the right calf. The anterior end of the ulcer had healed when I saw her, and beyond the posterior horn the skin was thickened and somewhat warty in consistency. The epileptic tendency of the patient was not disclosed to me at the time when I first saw her, and I regret to say that the correct diagnosis at first escaped me. Only sometime subsequently when I remembered the father's history did I make inquiry and ascertained that the little girl also suffered from epilepsy and thus arrived at the truth.

The second case I saw at the Royal Free Hospital; the patient was a woman who had been admitted for curettage for some uterine condition, the nature of which is unimportant, and I was asked to see her for a cutaneous lesion on the leg. This consisted of an oval-shaped area occupying a large portion of the outer side of the left calf. The dimensions were about six inches in length and it was two inches wide at its broadest part. The lower two-thirds was practically all scar tissue from the healing of previous ulceration, it was pitted and pale, but not particularly characteristic in any way. The upper third, however, was thickened and warty, pus was oozing from a few points, and there was one place about the size of a thumbnail where the skin was obviously distended with fluid. There was also a single pale scar about the size of a penny on the other leg. Inquiry elicited the information that she had been taking with her food a special "salt" given her by her doctor for the prevention of fits. On analysis this " salt" proved to contain 20 per. cent. of sodium bromide, and she told me she consumed about two ounces of the salt per month, roughly three drachms of bromide, again roughly about five grains a day.

The third was a case sent to me by Dr. Edythe Lindsay of Aldershot. This patient, also a woman, had a single button-shaped lesion on the inner side of the left knee. She proved to be particularly interesting, for when I asked her if she had any other "spots" anywhere she told me she had something on her abdomen. On examination I found in the situation indicated several plaques of arsenical keratosis set in the midst of a large area of typical raindrop pigmentation. This naturally led to an inspection of her palms and soles, which were studded with arsenical warts and presented a surface very like that of a nutmeg grater. Further signs of chronic arsenical poisoning were exhibited by total loss of olfactory sensation and cramps in the limbs. Cross-examination of the patient elicited the fact that 23 years previously she had consulted the late Sir William Gowers, who had prescribed for her a mixture containing 5 grains of bromide of potassium, 3 minims of liquor arsenicalis in each dose. She had been taking it regularly ever since. No doubt the arsenic had been given in order to minimise the effects of the bromide, which it had done pretty successfully, but it is doubtful whether it would not have been better for the patient to have a bromide rash rather than chronic arsenical poisoning. On my advice she discontinued the arsenic, and within a month the arsenical warts round the sweat glands had sensibly diminished and so had the hyperkeratosis of the soles; on the other hand, the solitary bromide lesion had grown larger and looked more angry. This was additional evidence that the arsenic had largely counteracted the bromide in previous times.

Dther attempts have been made also to counteract the effects of bromide, for this is sometimes a matter of great importance to patients, but till recently not with great success. K. Hübschmann recommends calcium therapy on the ground that the calcium content of the blood is diminished in bromodermia. The results of this form of medication I am not able to state. Quite lately, however, my attention has been drawn by Dr. Semon ${ }^{1}$ to an American method of treatment, based on the idea that mass action by sodium chloride will drive out bromide from solution in the body fluids and promotes its excretion by the kidneys; he therefore administers common salt in doses of about a drachm once a day, and in the few cases in which he has tried this simple remedy he is much gratified with the results obtained. It is to be hoped that we have here found an effective remedy for bromism.

1 Stevens: Sodium Chloride in the Treatment of Bromism, Amer. Arch. for Derm. and Syph., 1925, xii., 525-2 7 . 